\title{
Electrochemical tuning and mechanical resilience of single-wall carbon nanotubes*
}

\author{
Shankar Ghosh, Pallavi V. Teredesai, and A. K. Sood ${ }^{\ddagger}$ \\ Department of Physics, Indian Institute of Science, Bangalore 560 012, India
}

\begin{abstract}
Single-wall carbon nanotubes (SWNTs) are fascinating systems exhibiting many novel physical properties. In this paper, we give a brief review of the structural, electronic, vibrational, and mechanical properties of carbon nanotubes. In situ resonance Raman scattering of SWNTs investigated under electrochemical biasing demonstrates that the intensity of the radial breathing mode varies significantly in a nonmonotonic manner as a function of the cathodic bias voltage, but does not change appreciably under anodic bias. These results can be quantitatively understood in terms of the changes in the energy gaps between the 1D van Hove singularities in the electron density of states, arising possibly due to the alterations in the overlap integral of $\pi$ bonds between the p-orbitals of the adjacent carbon atoms. In the second part of this paper, we review our high-pressure X-ray diffraction results, which show that the triangular lattice of the carbon nanotube bundles continues to persist up to $\sim 10 \mathrm{GPa}$. The lattice is seen to relax just before the phase transformation, which is observed at $\sim 10$ GPa. Further, our results display the reversibility of the 2D lattice symmetry even after compression up to $13 \mathrm{GPa}$ well beyond the $5 \mathrm{GPa}$ value observed recently. These experimental results explicitly validate the predicted remarkable mechanical resilience of the nanotubes.
\end{abstract}

\section{INTRODUCTION}

Carbon nanotubes have unique structural, mechanical, and electronic properties. It is, thus, not surprising that they are being studied very actively ever since their discovery [1] from a basic science point of view, as well as for potential applications [2] such as actuators [3], pressure [4] and flow [5] sensors, nanoscale electron devices [4], catalysts, light-weight batteries, and hydrogen storage. Electrons confined in one dimension behave fundamentally different than in three dimensions. In one dimension, electrons form a correlated liquid, called the Luttinger liquid, which has novel features like separation of spin and charge and a power law dependence of the resistance on the bias voltage and temperature. Recent experiments [3] suggest that signatures of Luttinger liquid are found in single-wall carbon nanotubes (SWNTs).

Carbon nanotubes have been shown to be excellent actuators where the actuating action has been attributed not to the intercalation of ions, but due to the electrochemical double-layer charging [4]. This, in turn, is expected to change the electronic structure of the nanotubes. Our motivation was to quantify the effect of the electrochemical biasing of the nanotubes on the electronic band structure over and above the doping. This has been achieved [6] by employing resonance Raman scattering (RRS), which probes both vibrational and electronic states. Raman spectra of SWNTs show two prominent bands: one near $\sim 180 \mathrm{~cm}^{-1}$ associated with the radial breathing mode (RBM) and the other near $1590 \mathrm{~cm}^{-1}$ attrib-

*Pure Appl. Chem. 74, 1489-1783 (2002). An issue of reviews and research papers based on lectures presented at the $2^{\text {nd }}$ IUPAC Workshop on Advanced Materials (WAM II), Bangalore, India, 13-16 February 2002, on the theme of nanostructured advanced materials.

${ }^{\ddagger}$ Corresponding author 
uted to the tangential mode (TM). The frequency of the radial mode depends inversely on the tube diameter and the van der Waals interaction between the tubes assembled in bundles. The resonant scattering process arises from the 1D van Hove singularities in the electronic density of states with energy gaps, which depend inversely on the tube diameter. Thus, the diameter-selective RRS has been effectively used to determine the mean diameter, width of the diameter distribution, and the van der Waals interaction between the nanotubes in a bundle [7].

The second part of our work is related to pressure effects on the stability of nanotube bundles. Hexagonal graphite has lowest internal energy and, hence, is the equilibrium structure of carbon at ambient conditions. Pressure-induced phase transitions under static and dynamic loading between the many allotropes of carbon-like cubic diamond, hexagonal diamond, graphite, fullerenes $\mathrm{C}_{60}$, and $\mathrm{C}_{70}$ and their polymeric and amorphous forms are of great scientific interest as well as have practical importance. Earlier, we used Raman spectroscopy to explore the pressure and temperature-induced orientational phase transitions in solid $\mathrm{C}_{60}$ and solid $\mathrm{C}_{70}$, [8-9], pressure-induced amorphization of solid $\mathrm{C}_{70}$ [10], and pressure-temperature-induced polymeric phases [11,12]. We will review our high-pressure studies on SWNT bundles carried out up to $26 \mathrm{GPa}$ using Raman spectroscopy $[13,14]$ and up to $13 \mathrm{GPa}$ using X-ray diffraction [15]. These experimental studies explicitly validate the predicted remarkable mechanical resilience of the nanotubes.

Before the main results mentioned above are presented, we shall introduce the geometrical structure of nanotubes and their electronic, vibrational, and mechanical properties.

\section{GEOMETRICAL STRUCTURE OF NANOTUBES}

An SWNT can be thought of as a graphene sheet rolled up into a cylinder with caps made up of $\mathrm{C}_{60}$ hemispheres. In general, SWNTs can be specified [16] in terms of the diameter, $d$, and chiral angle, $\theta$, as shown in Fig. 1. The chiral vector is defined as

$$
|\mathbf{O B}|=\frac{\sqrt{3}\left|\mathbf{C}_{\mathbf{h}}\right|}{d^{\prime}}
$$

which connects two crystallographically equivalent sites $\mathrm{O}$ and $\mathrm{A}$ on a $2 \mathrm{D}$ graphene sheet. $\mathbf{a}_{1}$ and $\mathbf{a}_{\mathbf{2}}$ are the unit vectors of the honeycomb lattice connecting two inequivalent sites; $n$ and $m$ are integers. A tube

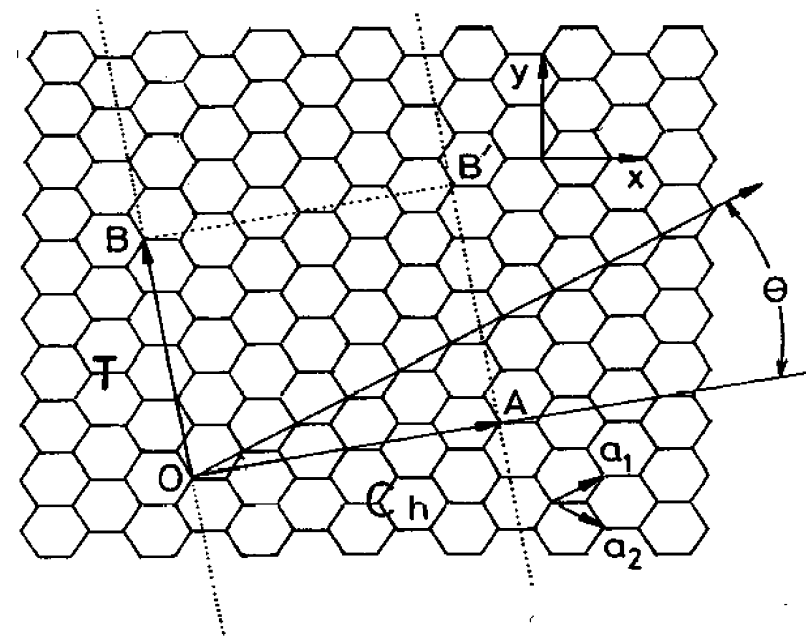

Fig. 1 The chiral vector $\mathbf{C}_{\mathbf{h}}=\mathbf{n} \mathbf{a}_{\mathbf{1}}+\mathbf{m} \mathbf{a}_{\mathbf{2}}$ is defined on the honeycomb lattice of carbon atoms by unit vectors $\mathbf{a}_{\mathbf{1}}$ and $\mathbf{a}_{\mathbf{2}}$ and the chiral angle $\theta$ with respect to the zigzag axis. Also, the lattice vector $\mathbf{O B}=\mathbf{T}$ of 1D tubule unit cell is shown. The diagram is constructed for $(n, m)=(4,2)$ (adapted from ref. 16). 
can be imagined being rolled up in such a way that its circumference is along $\mathbf{C}_{\mathbf{h}}$ and the tube axis is along the perpendicular direction to $\mathbf{C}_{\mathbf{h}}$. In Fig. 1, point A is joined to $\mathrm{O}$, and $\mathrm{B}^{\prime}$ is joined to $\mathrm{B}$. All other lattice sites can be mapped by fundamental lattice displacements using $\mathbf{a}_{\mathbf{1}}$ and $\mathbf{a}_{\mathbf{2}}$. The unit cell of each SWNT can be defined in terms of the integers $n$ and $m$ by specifying a unit cell lattice vector $\mathbf{O B}$ along the cylindrical axis of the tube. This vector can be constructed in a $2 \mathrm{D}$ graphene sheet by connecting the origin $\mathrm{O}$ to the first point in the lattice in the direction perpendicular to $\mathbf{C}_{\mathbf{h}}$ and has a magnitude given by [16].

$$
|\mathbf{O B}|=\frac{\left|\mathbf{C}_{\mathbf{h}}\right|}{\sqrt{3} d^{\prime}}
$$

if $n-m$ is not a multiple of $3 d^{\prime}$; or

$$
d=\frac{\mathbf{C}_{\mathbf{h}}}{\pi}=\frac{\left.d_{c-c} \sqrt{3\left(m^{2}+m n+n^{2}\right.}\right)}{\pi}
$$

if $n-m$ is a multiple of $3 d^{\prime}$, where $d^{\prime}$ is the highest common divisor of $n$ and $m$.

In terms of the integers $n$ and $m$, the tube diameter is given by [16] where $d_{c-c}$ is the nearest neighbor $\mathrm{C}-\mathrm{C}$ distance (= $1.421 \AA$ in graphite). The chiral angle $\theta$ is given by

$$
\theta=\tan ^{-1}[\sqrt{3} m /(m+2 n)]
$$

Depending on the $\theta$ value, SWNTs can be classified into three categories. When $\theta=30^{\circ}$, the tube is termed an armchair tube, whereas for $\theta=0^{\circ}$, the tube is called a zigzag tube. If $0^{\circ}<\theta<30^{\circ}$, it corresponds to a chiral tube implying that there is no symmetry plane perpendicular to the tube axis. The names "armchair" and "zigzag" are suggestive of the arrangement of carbon atoms along the circumference. Each SWNT can be specified by the pair of integers $(n, m)$ :

Armchair tube $(n, n): n \neq m$

Zigzag tube $\quad(n, 0): m \neq 0$

Chiral tube $\quad(n, m): n \neq m \neq 0$

It was shown that the SWNTs arrange themselves in $2 \mathrm{D}$ triangular lattice to form bundles of aligned SWNTs, which are energetically most favorable structures [17]. The tubes in a bundle interact with each other by van der Waals interaction as between the planes along the $c$-axis in graphite. According to the calculations done by Tang et al. [18], the nearest intertubular gap is $3.12 \AA$ at normal pressure compared to $3.35 \AA$ for the (002) graphite spacing.

\section{ELECTRONIC STRUCTURE OF NANOTUBES}

SWNTs can be semiconducting or metallic depending on the diameter and the chirality of the tubes. All zigzag tubes $(n, 0)$ are metallic when $n$ is divisible by 3 . Similarly, all armchair tubes are metallic. We can understand all this in a simple-minded, quantitative way from zone-folding effects. The density of states (DOS) of SWNTs calculated by tight binding approximation does not consist of one smooth band, as in bulk semiconductors, but shows a number of side-bands with spikes around the Fermi level separated from each other. These spikes are called the van Hove singularities. The side-bands represent 1D channels for conduction along the nanotube. The side-bands and their sharp onsets have been observed in their tunneling DOS obtained from scanning tunneling spectroscopic measurements [21]. For larger-diameter tubes, the singularities move close together and merge, thereby making the DOS resemble that of a graphene layer. On the other hand, for smaller-diameter nanotubes, the singularities are well separated especially near the Fermi level and can provide initial and final states for an RRS process. In a simplified picture, neglecting all the curvature effects and taking into account the linear 
dispersion of $\pi$ and $\pi^{*}$ bands of graphene, the energy gap between $i^{\text {th }}$ van Hove singularity in valence and conduction bands for semiconducting tubes is given by [11] $E_{i i} \mathrm{~S}=2 i \gamma_{0} d_{c-c} l d(i=1,2,3, \ldots)$. For metallic tubes, the separation between the van Hove singularities is 3 times higher given by $E_{i i}{ }^{\mathrm{M}}=6 i \gamma_{0} d_{c-c} / d$. In the case of metallic tubes, the linear crossing of $\pi$ and $\pi^{*}$ bands near the Fermi level yields a small constant DOS leading to sharp van Hove singularities at energies away from the Fermi energy. On the other hand, for semiconducting tubes, the DOS at Fermi energy is zero with sharp van Hove singularities away from the Fermi energy. Kataura et al. [19] showed that when all $q$ values within the Brillouin zone are included in the calculation of the van Hove singularities in the DOS of nanotubes, there are deviations from the simplified relations, $E_{i i} \mathrm{~S}=2 i \gamma_{0} d_{c-c} / d$ for semiconducting tubes and $E_{i i}{ }^{\mathrm{M}}=6 i \gamma_{0} d_{c-c} / d$ for metallic tubes. It was seen that $E_{22}$ is not exactly equal to $2 E_{11}$ and so on. Importantly, it is seen that when the isolated tubes are brought together to form a bundle, the inter-tube van der Waals interaction results in a finite gap $E_{\mathrm{vdw}}$, making the energy gap [20]

$$
E_{i i}^{\mathrm{s}} \cong \frac{2 i \gamma_{0} d_{c-c}}{d}+E_{\mathrm{vdw}}
$$

where $E_{\mathrm{vdw}} \sim 0.2 \mathrm{eV}[21]$.

\section{VIBRATIONAL PROPERTIES OF NANOTUBES}

As for electronic structure, the simplest way to calculate the phonon modes for carbon nanotubes is based on zone-folding of the phonon dispersion curves for a 2D graphene layer. Periodic boundary conditions are applied along the circumferential direction of the SWNT. Hence, the allowed values of wave vectors consist of a set of discrete wave vectors obeying $q=\frac{2 \pi p}{\left|\mathbf{C}_{\mathbf{h}}\right|}=\frac{2 p}{d} \quad(p=1,2, \ldots)$. The phonon frequencies can be read out from the dispersion relation of a graphene sheet corresponding to $q=2 p / d$ $(p=1,2, .$.$) , assuming that the dispersion relation remains unchanged by rolling the sheet into a closed$ tube. The observed multiply split peaks near $1590 \mathrm{~cm}^{-1}$ correspond to the $E_{2 g}(2)$ mode in graphite called as the tangential mode. These modes are associated with motion of carbon atoms along the tube axis as well as along the circumferential direction. According to polarization dependent studies carried out by Sun et al. [22], the assignment can be done as $1531 \mathrm{~cm}^{-1}$ as $E_{1 g}, 1553$ and $1568 \mathrm{~cm}^{-1}$ as $E_{2 g}$, $1594 \mathrm{~cm}^{-1}$ as unresolved doublet $A_{1 g}+E_{2 g}$ and $1606 \mathrm{~cm}^{-1}$ as $E_{2 g}$ symmetry. The tangential mode frequencies show weak dependence on the diameter of the tubes [23].

Apart from the tangential mode, there exists breathing mode of $A_{1 g}$ symmetry, which consists of a collective motion of all the atoms of the tube in the radial direction. This mode is termed a radial breathing mode (RBM). The RBM is a characteristic of the tubular structure of SWNT and cannot be explained on the basis of a zone-folding scheme. Theoretical calculations carried out by Bandow et al. [23] using the force constant model show that the frequency of the RBM $\left(A_{1 g}\right)$ depends inversely on the tube diameter and is independent of the helicity of the tubes as given by

$$
\omega_{R}\left(\mathrm{~cm}^{-1}\right)=\frac{223.75}{d(\mathrm{~nm})}
$$

Later, generalized tight binding molecular dynamics (GTBMD) calculations done by Venkateswaran et al. [24] pointed out that the RBM frequency gets blue-shifted due to the inter-tube interactions by $\sim 14 \mathrm{~cm}^{-1}$. From the ab initio calculations done by Hulman et al. [25], the RBM frequency for a tube in a bundle was given by

$$
\omega_{R}\left(\mathrm{~cm}^{-1}\right)=\frac{C_{1}}{d(\mathrm{~nm})}+C_{2} d(\mathrm{~nm})
$$


where, $C_{1} \sim 234 \mathrm{~cm}^{-1}-\mathrm{nm}, d$ is the diameter of the tube in $\mathrm{nm}$ and $C_{2}$ is a scaling constant for inter-tube interaction, which depends weakly on the bundle diameter. Typically, $C_{2} \sim 12 \mathrm{~cm}^{-1} / \mathrm{nm}$.

It has been observed that the line shape of the tangential mode is very different for semiconducting and metallic tubes [26]. For a tube with diameter $d=1.49 \pm 0.2 \mathrm{~nm}$, the laser beam is in resonance with the $E_{22}{ }^{\mathrm{S}}$ transition for semiconducting nanotubes for $E_{\text {laser }}=1.49 \mathrm{eV}$, while for $E_{\text {laser }}=1.96 \mathrm{eV}$, most of the nanotubes are in resonance with the $E_{11}{ }^{\mathrm{M}}$ transition for metallic nanotubes with some nanotubes on the high end of the diameter distribution being in resonance with the $E_{33} \mathrm{~S}_{(d)}$ transition for semiconducting nanotubes. For $E_{\text {laser }}=1.92 \mathrm{eV}$, both semiconducting and metallic nanotubes participate in the resonance.

\section{MECHANICAL PERFECTION OF SWNTS}

SWNTs are one of the most flexible fibers known to mankind. SWNTs can be 100 times stronger than steel while they are one-sixth of its weight [27]. Pressing on the tip of the nanotube will cause it to bend without damage to the tip or the whole SWNT. When the force is removed, the tip of the nanotube recovers to its original shape [28]. SWNTs, therefore, appear to be ideal tips for scanning probe microscopes since they are not only very small but also can survive crashes with the sample surface [29]. The Young's modulus of SWNTs lies close to $1 \mathrm{Tpa}$, and the maximum tensile strength is $30 \mathrm{GPa}$ [30]. Ijima et al. [31] reported high-resolution electron microscope (HREM) observations and atomistic simulations of the bending of SWNTs under mechanical stress. It was shown that the bending, in spite of having multiple kinks and highly strained tube regions, is fully reversible up to very large bending angles as high as $110^{\circ}$. The molecular dynamic (MD) simulations of Yakobson et al. [32] showed that SWNTs subjected to large deformations switch into different morphological patterns where each shape corresponds to an abrupt release of energy and a singularity in the stress-strain curve. These deformations are reversible on decompression.

SWNTs are highly anisotropic uniaxial structures [33,34]. The tube is extremely rigid along the tube axis as expected because any distortion along the tube axis is equivalent to the in-plane distortion of graphite. It was proposed by Reich et al. [35] that the strain resulting from hydrostatic pressure in general should be different for the length and the circumference of the tube. Consequently, under hydrostatic pressure, one should expect different pressure slopes for modes vibrating parallel and perpendicular to the axis. The calculated strain component along the circumferential direction is almost three times higher than that along the tube axis [35]. Thus, the tubes can be easily distorted perpendicular to their axis and can result in the contact area being flattened on bringing two nanotubes closer [36] and in the observed collapsed structure at some places in the nanotube bundle [37].

\section{ELECTROCHEMICAL TUNING OF BAND STRUCTURE OF SWNTS}

Carbon nanotubes have also been shown to be excellent actuators [4] where the actuating action has been attributed not to the intercalation of ions, but owing to the electrochemical double layer charging. This, in turn, is expected to change the electronic structure of the nanotubes. Our motivation was to quantify the effect of the electrochemical biasing of the nanotubes on the electronic band structure over and above the doping. This has been achieved [6] by employing resonance Raman scattering (RRS), which probes both vibrational and electronic states. SWNT bundles formed one electrode in an electrochemical cell, and platinum was used as a counterelectrode. The electrolyte used was $0.05 \mathrm{M} \mathrm{LiClO}_{4}$. In situ micro-Raman scattering experiments were carried out in a back-scattering geometry. Raman spectra were excited using $514.5 \mathrm{~nm}\left(E_{\mathrm{L}}=2.41 \mathrm{eV}\right)$ and $457.9 \mathrm{~nm}\left(E_{\mathrm{L}}=2.71 \mathrm{eV}\right)$ radiations from an argon ion laser. DILOR-XY spectrometer equipped with liquid nitrogen-cooled charge-coupled device detector was used. 
Figure 2 shows the Raman bands due to RBM and TM (in the inset) at zero bias (curve a), and at a bias of $-0.08 \mathrm{~V}$ (curve b) using $E_{\mathrm{L}}=2.41 \mathrm{eV}$. For the RBM, the solid lines represent the data and the dotted lines are the calculated line shapes (to be explained later). Figure 3 shows the integrated intensi-

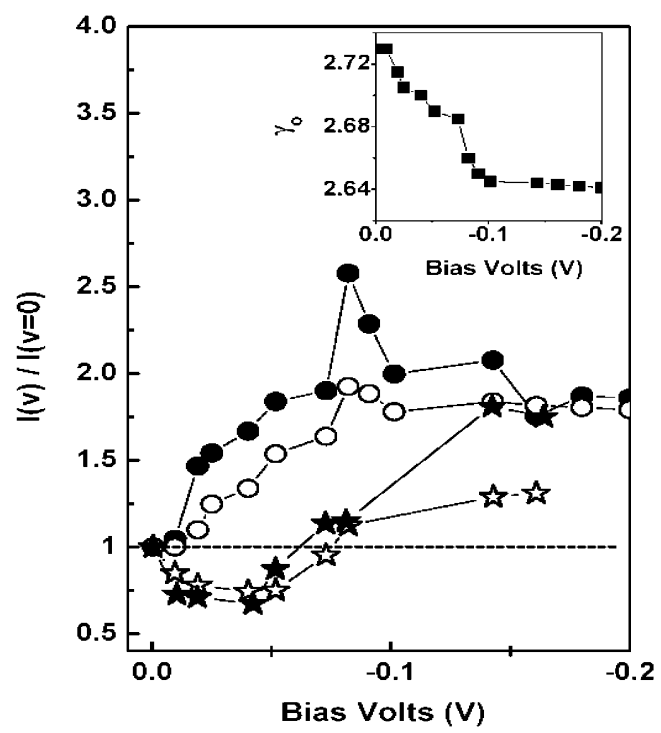

Fig. 2 Experimental Raman spectra (solid lines) of the RBM at zero bias (a) and $-0.08 \mathrm{~V}$ (b) for $E_{\mathrm{L}}=2.41 \mathrm{eV}$. The dotted lines show the calculated line shapes as explained in the text. Inset shows the observed Raman spectra in the TM regime at zero bias (open circles) and at $-0.08 \mathrm{~V}$ (solid line) [6].

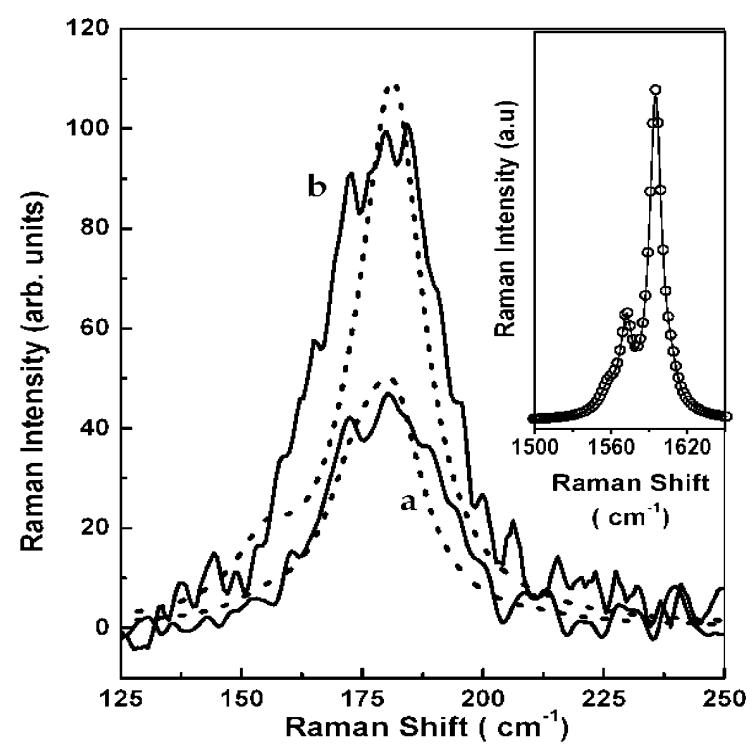

Fig. 3 Variation of the integrated intensities of the modes normalized with respect to the zero bias spectra for RBM (circles for $E_{\mathrm{L}}=241 \mathrm{eV}$, stars for $E_{\mathrm{L}}=271 \mathrm{eV}$ ). Solid symbols are experimental data. Open symbols correspond to the calculated intensities with (v) as the only adjustable parameter. The voltage-dependence of (v) is same for both $E_{\mathrm{L}}$. Inset shows variation of the overlap integral $\gamma_{0}$, with the applied bias voltage (v). [6] 
ties of the Raman bands by filled circles $\left(E_{\mathrm{L}}=2.41 \mathrm{eV}\right)$ and filled stars $\left(E_{\mathrm{L}}=2.71 \mathrm{eV}\right)$. The intensities in Fig. 3 have been normalized with respect to the intensities under open-circuit condition. Most interestingly, we see that on the application of the negative bias on the SWNT electrode with respect to the Pt counterelectrode, the intensity of the RBM shows significant nonmonotonic changes, being very different for the two exciting laser energies. For low-bias voltage (less than $0.05 \mathrm{~V}$ ) the intensity increases when $E_{\mathrm{L}}=2.41 \mathrm{eV}$, in sharp contrast to the case when $E_{\mathrm{L}}=2.71 \mathrm{eV}$. These changes in intensity are reversible on removal of the bias. There is no shift in the frequency of RBM and TM with the applied bias. This can be understood on the basis that at such low-bias voltages, the charge transfer per carbon atom is very small.

We now discuss the nonmonotonic variation of the intensity of the RBM as a function of cathodic bias (cf. Fig. 3). This variation can be understood as due to the changes in the resonance Raman condition associated with the bias-dependent effects on the electronic structure of nanotubes. The electronic excitations in the nanotubes participating in Raman scattering are the transitions of electrons from the $i^{\text {th }}$ valence band singularity $\left(v_{i}\right)$ to the $j^{\text {th }}$ conduction band singularity $\left(c_{j}\right)$. However, symmetry reasons allow only $v_{i} \rightarrow c_{i}$. Within the tight binding approximation, the energy gap between $v_{i}$ and $c_{i}$ van Hove singularities is given by eq. 2 . Resonant enhancement occurs both for the incident (incoming resonance), as well as the scattered photons (outgoing resonance).

The Raman line shape can now be written as

$$
I(\omega)=\sum_{\omega_{p h}}\left(A \sum_{i=1}^{15} \sum_{d} \frac{1}{d^{2}} \frac{p(d)}{\left[\left(E_{i i}-E_{\mathrm{L}}\right)^{2}+\Gamma_{e}^{2}\right]\left\{\left[E_{i i}-\left(E_{\mathrm{L}}-h \omega_{p h}\right)\right]^{2}+\Gamma_{e}^{2}\right\}}\right) \frac{\Gamma}{4\left(\omega-\omega_{p h}\right)^{2}+\Gamma^{2}}
$$

where $\omega_{p h}$ is the phonon frequency, $\Gamma$ (full width at half-maximum) is the phonon damping constant. The term in the parenthesis is the Stokes Raman cross-section from a collection of nanotubes with diameter distribution $p(d), 1 / d^{2}$ is the contribution from the joint density of electronic states. $\Gamma_{e}$ is the broadening parameter for the electronic bands. The distribution of diameters was taken to be a Gaussian centered at $d_{\mathrm{o}}$ :

$$
p(d)=e^{-\left(d-d_{0}\right)^{2} / 2 \sigma^{2}},
$$

taking $\sigma$ to be $0.08 \mathrm{~nm}$. The parameter $A$ contains all three matrix elements related to electron-radiation and electron-phonon interaction Hamiltonians, which are assumed to be constant [38]. The RBM frequencies have a diameter dependence given by eq. 3 . The second term arises due to the van der Waals intertube interactions. The tangential modes have no dependence on $d$. The resonance width $\Gamma_{e}=0.05 \mathrm{eV}$ and $\Gamma=15 \mathrm{~cm}^{-1}$ were found to be appropriate to match the calculated and experimental spectra. For zero bias conditions, the value of $\gamma_{0}$ obtained from the best fit of the data $\left(E_{\mathrm{L}}=2.41 \mathrm{eV}\right)$ to eq. 4 was found to be $2.73 \mathrm{eV}$, which is well in accordance with the reported values [25,37,38]. The calculated line shape is shown in Fig. 2 by the solid line (curve a), which fits the experimental data well. The constant $A$, obtained by normalizing the observed $1594 \mathrm{~cm}^{-1} \mathrm{TM}$ peak intensity for zero bias condition with the calculated one, was kept constant subsequently for line shape analysis of the RBM at all bias voltages. Although the summation over the order of the electronic transitions was done for $i=1$ to 15 , the main contribution to the Raman intensity comes from the $i=4$ electronic transition $\left(E_{44} \sim 2.3 \mathrm{eV}\right)$.

Experimental data for the finite cathodic bias were fitted using eqs. 4 , with only one adjustable parameter, $\gamma(\mathrm{v})$. The dotted line in curve $\mathbf{b}$ of Fig. 2 shows the calculated line shape with $\gamma(\mathrm{v}=-0.08 \mathrm{~V})$ for $E_{\mathrm{L}}=2.41 \mathrm{eV}$. The agreement between experimental and calculated line shapes is reasonable. The voltage-dependence of $\gamma_{0}$ obtained from analyzing the Raman data corresponding to $E_{\mathrm{L}}=2.41 \mathrm{eV}$ is completely consistent with that obtained from the Raman data using $E_{\mathrm{L}}=2.71 \mathrm{eV}$. The calculated total intensities for different bias voltages are shown in Fig. 3 as open circles for $E_{\mathrm{L}}=2.41 \mathrm{eV}$ and as open stars for $E_{\mathrm{L}}=2.71 \mathrm{eV}$. The trends in the nonmonotonic variation in Raman intensity as a function of the 
applied cathodic bias are satisfactorily reproduced by the calculations for both the values of $E_{\mathrm{L}}$. Figure 3 (inset) shows the variation of $\gamma(\mathrm{v})$ with the applied voltage.

It is interesting that although the RBM exhibits a nonmonotonic variation in the intensity as a function of bias voltage, the TM does not. This can be understood from the fact that the $\omega_{p h}$ of the tangential mode does not have a diameter dependence. A small change in $E_{i i}$ affects the RBM much more than the TM.

We now offer qualitative reasons to understand why $\gamma_{0}$ can change under cathodic biasing of the nanotubes. With negative bias applied to the SWNT, the cations $\mathrm{Li}^{+}$form a space-charge double layer around the nanotubes which affect the overlap integral of the $\pi$ orbitals on adjacent carbon atoms separated by $d_{c c} \sim 0.142 \mathrm{~nm}$. This effect should naively depend on the size of cations. This is indeed the case in our experiments. The effect (seen in Fig. 3) using $\mathrm{Li}^{+}$(ionic size $\sim 0.12 \mathrm{~nm}$ ) is much larger than $\mathrm{K}^{+}$(ionic size $\sim 0.28 \mathrm{~nm}$ ) (data not shown). We believe that large ionic size is also responsible for not seeing the effect under anodic bias (ionic size of ions $\mathrm{CIO}^{-} \sim 0.8 \mathrm{~nm}, \mathrm{Cl}^{-} \sim 0.36 \mathrm{~nm}$ ). More theoretical work is needed to quantify this novel effect.

\section{PRESSURE EFFECTS}

We have carried out high-pressure Raman studies on SWNT bundles up to 25.9 Gpa [13,14]. The intensity of the radial modes decreases more drastically as compared to that of the tangential modes. The former could be followed up in pressure runs until $3 \mathrm{GPa}$. The most intriguing observation is the anomalous pressure behavior of the $1594 \mathrm{~cm}^{-1}$ tangential mode between 10 and $16 \mathrm{GPa}$. The mode softens as a function of pressure. The pressure dependence of intensity, peak position, and line width are reversible on decompression.

Our results are contradictory to the measurements done by Peters et al. [40], which showed a phase transition at $1.7 \mathrm{Gpa}$, at which SWNTs undergo a structural transformation from triangular to monoclinic lattice. X-ray diffraction study of SWNTs under pressure [18] suggested vanishing of the triangular lattice at $\sim 1.5 \mathrm{GPa}$ and its regeneration if unloaded from less than $4 \mathrm{GPa}$. Beyond $5 \mathrm{GPa}$, these X-ray results indicated an irreversible change, in total contrast to our Raman results. So, in order to find a consistent interpretation to our results as well as several other experiments, we did in situ highpressure X-ray diffraction studies on SWNT bundles using Synchrotron Radiation source SPring8 [15].

Figure 4 shows variations in the intensity of the diffraction pattern corresponding to the $2 \mathrm{D}$ triangular lattice as a function of pressure in increasing as well as decreasing pressure runs. On increasing the pressure, the SWNT diffraction line vanishes beyond $10 \mathrm{GPa}$. On decompression, from 13 to $7 \mathrm{GPa}$, the diffracted intensity corresponding to the triangular symmetry reappears and regains almost full initial intensity on complete release of pressure. The vanishing intensity of the diffraction profile indicates a structural-phase transition. Since no new diffraction lines were observed across this pressure, there are two possibilities: (i) the new structure is such that it does not have any strong Bragg reflections and (ii) the translational coherence of the nanotubes in the bundle is lost.

According to Chesnokov et al. [35], SWNTs undergo irreversible graphitization at 9 GPa. Also, the calculated diffraction patterns by Sharma et al. [41], with totally circular and ideal hexagonal tubes, show that the intensity of the diffraction line is not affected significantly due to faceting. Recent molecular dynamic calculations [24,33] suggest that SWNTs are faceted even at ambient pressures. Thus, we rule out the possibility of uniform faceting/or uniform flattening of the tubes related to the structural transition at $\sim 10 \mathrm{GPa}$. The complete loss of the low-angle diffraction line due to the triangular lattice of the SWNT at $10 \mathrm{GPa}$ and its retrieval on decompression clearly demonstrates a reversible loss of translational coherence. We emphasis that our results are in sharp contrast to those of Tang et al. [16], who report an irreversible loss of triangular lattice when compressed above $4 \mathrm{GPa}$.

Figure 5 shows the variation of the d-spacing of various diffraction lines with pressure. The d-spacing corresponding to the diffraction from the (10) planes of triangular lattice shown by circles (topmost curve) are shown along with the lines due to diffraction from the gasket shown by diamond 


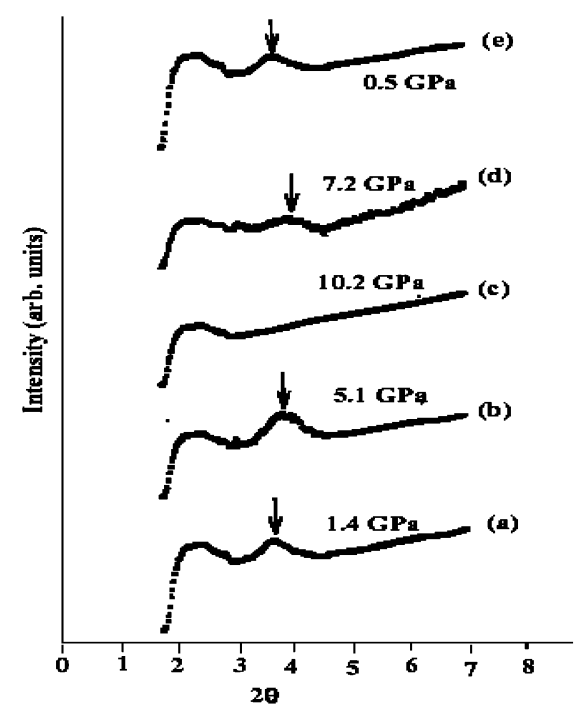

Fig. 4 Variations in the intensity of the diffraction pattern corresponding to 2D triangular lattice as a function of pressure. (a) to (c) are for increasing pressure run, whereas (d) and (e) are for the decreasing pressure run [15].

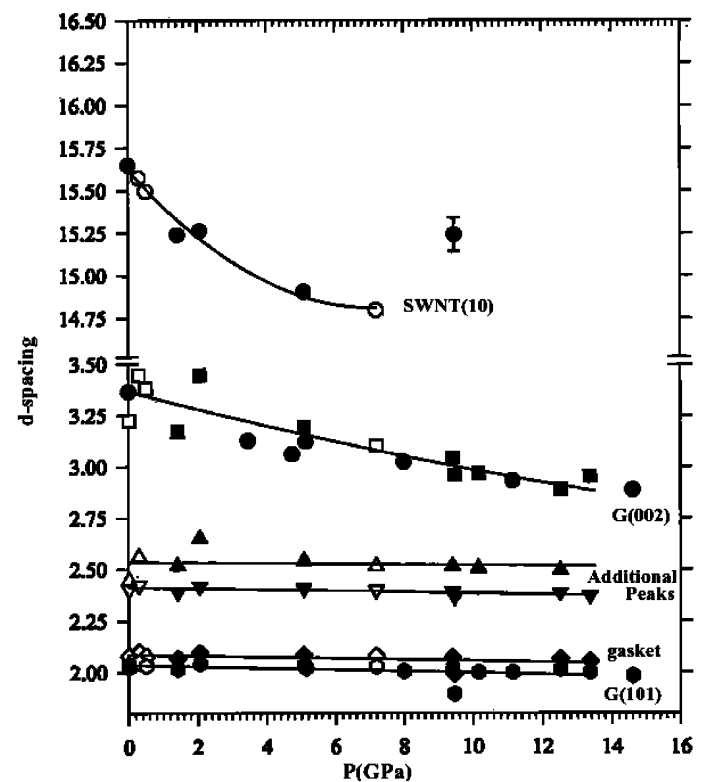

Fig. 5 Variation of the d-spacing of various diffraction lines with pressure [15].

symbols. Thermal gravimetric analysis of our SWNT sample shows existence of $8 \%$ graphite in the sample, and it is this graphite that gives rise to the two diffraction lines with d-spacings, marked by $\mathrm{G}(101)$ and $\mathrm{G}(002)$ shown by hexagons and circles, squares respectively. Figure 5 shows two additional lines marked by triangular symbols corresponding to the diffraction from SWNT bundles. The solid (open) symbols are for increasing (decreasing) pressure runs. Up to $\sim 8 \mathrm{GPa}$, we see a systematic com- 
pression as displayed by the reduction of d(10) of SWNT as a function of pressure. No sudden change in the variation of $\mathrm{d}$-spacing was observed at $\sim 1.7 \mathrm{GPa}$. The variation of the $2 \mathrm{D}$ triangular lattice parameter "a" with pressure can be fitted to the one-dimensional analog of the Murnaghan equation [42],

$$
\frac{a}{a_{o}}=\left[\left(\frac{\beta^{\prime}}{\beta_{0}}\right) P+1\right]^{\frac{-1}{\beta^{\prime}}}
$$

where $\beta_{0}$ is the bulk modulus and $\beta^{\prime}$ is the pressure derivative of bulk modulus. Fitting of the above equation to the data gives $\beta_{0}=43( \pm 4) \mathrm{GPa}$ and $\beta^{\prime}=33( \pm 3)$. Comparing these values to that for $c$-axis compression in graphite [43], $\beta_{0}=35.7 \mathrm{GPa}$ and $\beta^{\prime}=10.8$, one can say that up to $\sim 8 \mathrm{GPa}$, SWNTs are somewhat less compressible than graphite along $c$-axis. We calculated bulk modulus of the SWNT bundles from the formula $\beta=-\mathrm{V}(\Delta P / \Delta V)$, where $V$ is the unit cell volume of SWNT bundle. We calculated $V$ taking lattice constants of the triangular SWNT lattice as "a" and "b" and taking "c" to be $\sqrt{ } 3 d_{c-c}$. Then, the slope $\Delta P / \Delta V$, is found by extrapolating the $P-V$ curve by a quadratic fit up to zero pressure. Then, multiplying this zero-pressure slope with the zero-pressure unit cell volume, we get the zero-pressure bulk modulus. Assuming the tube axial compression as that of the graphite basal plane, the low-pressure bulk modulus for SWNT was then found to be $\sim 34 \mathrm{GPa}$.

Recently, Reich et al. [44] have carried out first principle calculation of the bulk and linear modulii of carbon nanotube bundles and individual tubes. They have used local-density approximation of density functional theory. The equation of state obtained from fits to the calculated total energy using Vinet's equation [45] agrees remarkably well with our data. Vinet's equation is given by

$$
\begin{aligned}
& P=\left[3 \beta_{0}(1-x) / x^{2}\right] \exp [\eta(1-x)] \\
& \eta=\frac{3}{2}\left(\beta^{\prime}-1\right) \\
& x=\left(\frac{V}{V_{\circ}}\right)^{1 / 3}
\end{aligned}
$$

The values obtained using Vinet's equation are $\beta_{0}=37 \mathrm{GPa}, \beta^{\prime}=11$. It may be noted that the corresponding calculated values for individual nanotubes are $\beta_{0}=230 \mathrm{GPa}$ and $\beta^{\prime}=4.5$. The calculations also reveal that the hexagonal distortion of the $2 \mathrm{D}$ triangular lattice of nanotubes $(6,6),(10,0)$, and $(8,4)$ having diameter $\sim 8 \AA$ is less than $5 \%$ even up to a pressure of $8.5 \mathrm{GPa}$. However, at a pressure of $\sim 10 \mathrm{GPa}$, the armchair nanotubes collapse to flat ellipses.

Figure 5 shows another remarkable feature; d(10) of SWNT bundles relaxes to a higher value at $\sim 9 \mathrm{GPa}$, just before the material undergoes the phase transformation. The variation of the $\mathrm{d}(002)$ and d(101) diffraction lines of graphite do not show any relaxation effect at $\sim 9 \mathrm{Gpa}$, establishing that the SWNTs undergo structural changes involving morphology of the tubes. This is consistent with our earlier observation of "softening" [14] around 10 GPa in tangential mode frequency in high-pressure Raman experiments. This can be attributed to the formation of kinks and fins as described in the MD simulations [30,31] of Ijima et al. around 1 Gpa.

In summary, we have reviewed structural, electronic, vibrational, and mechanical properties of SWNT bundles. Our resonance Raman studies have revealed that the overlap integral between the $\pi$-electrons on the neighboring carbon atoms in the nanotube can change under electrochemical negative bias, thereby changing the energy gaps between the van Hove singularities in valence and conduction bands. In another study, we have also shown that SWNT bundles lose their translational coherence at $\sim 10 \mathrm{GPa}$ which reverts on decompression. These high-pressure Raman and X-ray diffraction experiments explicitly reveal the remarkable mechanical resilience of the nanotube bundles. It is certain that a lot more needs to be done in future to understand the fascinating properties of the nanotubes. 


\section{ACKNOWLEDGMENTS}

A.K.S. thanks the Department of Science and Technology, India for financial assistance. P.V.T. thanks CSIR, India for financial assistance. The work on nanotubes was done on the high quality of samples of nanotubes supplied by Prof. C. N. R. Rao. The high-pressure X-ray diffraction studies were done in collaboration with the BARC group of Dr. S. M. Sharma at Mumbai. We thank all our collaborators.

\section{REFERENCES}

1. S. Iijima. Nature (London) 354, 56 (1991).

2. C. N. R. Rao, B. C. Satishkumar, A. Govindraj, M. Nath. ChemPhysChem 2, 78 (2001).

3. R. Egger. Phys. Rev. Lett. 83, 5547 (1999).

4. R. H. Baughman, C. Cui, A. A. Zakhidov, Z. Iqbal, J. N. Barisci, G. M. Spinks, G. G. Wallace, A. Mazzoldi, D. De Rossi, A. G. Rinzler, O. Jaschinski, S. Roth, M. Kertesz. Science 284, 1340 (1999).

5. P. Kral and M. Shapiro. Phys. Rev. Lett. 86, 131 (2001).

6. S. Ghosh, A. K. Sood, C. N. R. Rao. J. Appl. Phys. (15 July 2002).

7. T. C. Charlier and Ph. Lambin. Phys. Rev. B 57, R15037 (1998).

8. N. Chandrabhas, M. N. Shashikala, D. V. S. Muthu, A. K. Sood, C. N. R. Rao. Chem. Phys. Lett. 197, 319 (1992); N. Chandrabhas, K. Jayram, D. V. S. Muthu, A. K. Sood, R. Seshadri, C. N. R. Rao. Phys. Rev. B: Rapid Commun. 47, 10963 (1993); S. K. Ramasesha, A. K. Singh, R. Seshadri, A. K. Sood, C. N. R. Rao. Chem. Phys. Lett. 220, 203 (1994).

9. A. K. Sood, N. Chandrabhas, D. V. S. Muthu, Y. Hariharan, A. Bharathi, C. S. Sunder. Philos. Mag. B 70, 347 (1994).

10. N. Chandrabhas, A. K. Sood, D. V. S. Muthu, C. S. Sunder, A. Bharathi, Y. Hariharan, C. N. R. Rao. Phys. Rev. Lett. 73, 3411 (1994).

11. C. S. Sunder, P. Ch. Sahu, V. S. Sastry, G. V. N. Rao, V. Sridharan, M. Premila, A. Bharathi, Y. Hariharan, T. S. Radhakrishnan, D. V. S. Muthu, A. K. Sood. Phys. Rev. B 53, 8180 (1996).

12. M. Premila, C. S. Sunder, P. Ch. Sahu, A. Bharathi, Y. Hariharan, D. V. S. Muthu, A. K. Sood. Solid State Commun. 104, 237 (1997).

13. A. K. Sood, P. V. Teredesai, D. V. S. Muthu, R. Sen, C. N. R. Rao. Phys. Status Solidi B 215, 393-401 (1999).

14. P. V. Teredesai, A. K. Sood, D. V. S. Muthu, R. Sen, A. Govindaraj, C. N. R. Rao. Chem. Phys. Lett. 319, 296 (2000).

15. S. M. Sharma, S. Karmakar, S. K. Sikka, P. V. Teredesai, A. K. Sood, A. Govindaraj, C. N. R. Rao. Phys. Rev. B 63, 2054 (2001).

16. M. S. Dresselhaus, G. Dresselhaus, P. C. Eklund. Science of Fullerenes and Carbon Nanotubes, Chap. 19, Academic, NY (1996).

17. H. D. Sun, Z. K. Tang, J. Chen, G. Li. Solid State Commun. 109, 365 (1999).

18. J. Tang, L. C. Qin, T. Sasaki, M. Yudasaka, A. Matsushita, S. Ijima. Phys. Rev. Lett. 85, 1887 (2000).

19. M. J. Peters, L. E. McNeil, J. P. Lu, D. Kahn. Phys. Rev. B 61, 5939 (2000).

20. T. C. Charlier and Ph. Lambin. Phys. Rev. B 57, R15037 (1998).

21. P. Delaney, H. J. Choi, I. Ihm, S. G. Louie, M. L. Cohen. Nature (London) 391, 466 (1998).

22. H. D. Sun, Z. K. Tang, J. Chen, G. Li. Solid State Commun. 109, 365 (1999).

23. S. Bandow, S. Asaka, Y. Saito, A. M. Rao, L. Grigorian, E. Richter, P. C. Eklund. Phys. Rev. Lett. 80, 3779 (1998).

24. U. D. Venkateswaran, A. M. Rao, E. Richter, M. Menon, A. Rinzler, R. E. Smalley, P. C. Eklund. Phys. Rev. B 59, 10928 (1999).

25. H. Hulman, W. Plank, H. Kuzmany. Phys. Rev. B 63, R081406 (2001). 
26. Science and Application of Nanotubes, D. Tomanek (Ed.), p. 259, Kluwer/Plenum, NY (2000).

27. B. L. Yakobson and R. E. Smalley. Am. Scientist 85, 324 (1997).

28. H. Dai, J. H. Hafner, A. G. Rinzler, D. T. Colbert, R. E. Smalley. Nature 384, 147 (1996).

29. M.-F. Yu, B. S. Files, S. Arepalli, R. S. Ruo. Phys. Rev. Lett. 84, 5552 (2000).

30. S. Ijima, C. Brabec, A. Maiti, J. Bernholc. J. Chem. Phys. 104, 2089 (1996).

31. B. I. Yakobson, C. J. Brabec, J. Bernholc. Phys. Rev. Lett. 76, 2511 (1996).

32. C. Dekker. Physics Today 22 (1999).

33. C. Thomsen, S. Reich, A. R. Goni, H. Jantoljak, P. M. Rafailov, L. Loa, K. Syassen, C. Journet, P. Bernier. Phys. Status Solidi B 215, 435 (1999).

34. J. P. Lu. Phys. Rev. Lett. 79, 1297 (1997).

35. S. Reich, H. Jantoljak, C. Thomsen. Phys. Rev. B 61, 13389 (2000).

36. S. A. Chesnokov, V. A. Nalimova, A. G. Rinzler, R. E. Smalley, J. E. Fischer. Phys. Rev. Lett. 82, 343 (1999).

37. Y. Q. Zhu, T. Sekine, T. Kobayashi, E. Takazawa, M. Terrones, H. Terrones. Chem. Phys. Lett. 287, 689 (1998).

38. P. M. Rafilov, H. Jantoljak, C. Thomsen. Phys. Rev. B 61, 16179 (2000).

39. M. A. Pimenta. Phys. Rev. B 58, R16016 (1998).

40. M. J. Peters, L. E. McNeil, J. P. Lu, D. Kahn. Phys. Rev. B 61, 5939 (2000).

41. S. M. Sharma et al. Unpublished.

42. F. D. Murnaghan. Proc. Natl. Acad. Sci. USA 30, 244 (1944).

43. M. Han, H. Beister, K. Syassen. Phys. Rev. B 39, 12598 (1989).

44. S. Reich, C. Thomsen, P. Ordejon. Phys. Rev. B 65, 153407 (2002).

45. P. Vinet, J. H. Rose, J. Ferrantes, J. R. Smith. J. Phys.: Condens. Matter 1, 1941 (1989). 